\title{
OBSERVATIONS ON THE PREPARATION OF TOXIN-ANTITOXIN MIXTURE
}

\author{
P. G. HEINEMAN AND CHARLES R. HIXSON
}

From the Research Laboratories, United States Standard Products Company, Woodzoorth, Wis.

Immunization against diphtheria by toxin-antitoxin has been eminently successful, and this means that another milestone has been passed in the efforts to eradicate the disease. Successful immunization depends on proper balance of toxin and antitoxin. The toxin is the active agent in producing immunity, while the antitoxin enters into a more or less firm combination with the toxin, thus protecting the system against serious toxic reaction. If the combination between toxin and antitoxin were a firm one, as in many chemical compounds, the excess toxin alone would be of immunizing value. The addition of antitoxin merely enables the system to bear a larger amount of toxin than would be possible without this addition. It is imperative that an effective toxin-antitoxin mixture should be so balanced as to have a slight excess of unneutralized toxin. A completely neutralized mixture has less immunizing value than a slightly toxic one, and a mixture containing an excess of antitoxin is of still smaller value.

Directions for the preparation of the toxin-antitoxin mixture are given by the Hygienic Laboratory, in Washington. According to these directions, the following steps are to be taken:

1. The toxin must be aged for at least three months to insure relative stability.

2. The $L+$ dose of the toxin is determined against the standard antitoxin issued by the Hygienic Laboratory, and the potency of the lot of serum to be used is determined against the $\mathrm{L}+$ dose of the toxin.

3. A tentative mixture is prepared which is allowed to stand for 24 hours before testing; a toxin is used which contains at least three $\mathrm{L}+$ doses for one human dose, and the amount of antitoxin necessary to produce the desired result is calculated and mixed with the toxin.

4. Two tests are made. The first one consists of injecting subcutaneously one human dose into a guinea-pig weighing $250 \mathrm{gm}$. The second test consists of injecting 5 human doses subcutaneously into a similar guinea-pig. The mixture is properly balanced if the guinea-pig receiving one human dose shows no ill effect, while the one receiving 5 human doses develops paralysis not earlier than the tenth day after 
injection and dies subsequently within 35 days after injection. A toxinantitoxin mixture of this quality therefore contains considerably less than one minimum lethal dose of free toxin in 5 human doses. The excess toxicity is distinct, but not large enough to cause serious symptoms.

5. If the guinea-pigs die before the appointed time, the excess of toxin is too large and a further quantity of antitoxin has to be added. The mixture is then tested again. This procedure may have to be repeated several times before a satisfactory mixture is obtained. If by chance the mixture is overneutralized, addition of toxin is not advisable.

6. The toxin-antitoxin mixture is then filled in containers. The contents of several containers are tested for sterility and are injected into guinea-pigs in the same manner as before. After four weeks another pair of guinea-pigs is injected. The first pair is kept under observation for five weeks and the second pair for one week. If the reactions on the guinea-pigs are satisfactory, the mixture is ready for use. ${ }^{1}$

Aging of the toxin is necessary to guard against rapid deterioration with loss of potency. Unfortunately, all toxins do not deteriorate at the same rate. Some lose toxicity more rapidly than others. As a rule, a toxin, when not sufficiently aged, deteriorates more rapidly than does antitoxin, and in this case the toxin-antitoxin mixture soon loses potency. Toxins set aside for preparation of toxin-antitoxin mixture should therefore be tested for $\mathrm{L}+$ dose several times until the value remains reasonably constant.

The $\mathrm{L}+$ dose of a toxin is tested against the standard antitoxin issued by the Hygienic Laboratory. As a matter of fact, however, a certain antitoxin may give slightly different values when tested against $\mathrm{L}+$ doses of different toxins. This is readily understood when Ehrlich's theory of the structure of the toxin molecule is considered. According to this theory the toxin molecule is composed chiefly of the prototoxoid (nontoxic), the toxin (the truly toxic portion) and the toxone (causing postdiphtheritic paralysis). Antitoxin combines more firmly with prototoxoid than with toxin, and more firmly with toxin than with toxone. If, therefore, the prototoxoid fraction in a given toxin is larger than in another one, the former requires more antitoxin to leave one M L D unneutralized than the latter. Although the determination of the value of the antitoxin to be used against the

1 Since this paper went to press the directions given by the Hygienic Laboratory have been changed and the toxicity of the product is increased thercby. 
$\mathrm{L}+$ dose of the toxin may not be absolutely necessary, it is advisable and guards against overneutralization.

A test of the tentative mixture of toxin and antitoxin is not made before 24 hours have elapsed in order to allow toxin and antitoxin to combine. A period of 24 hours, however, does not seem to be sufficient. It is possible that a fairly firm combination of antitoxin with prototoxoid may obtain in 24 hours, but the combination between toxin and antitoxin is slower than the former and between toxone and antitoxin still slower. It follows that the death of guinea-pigs injected with five human doses of the mixture must be more and more deferred in proportion to the age of the mixture. After a certain period, which has not been determined and which probably varies in different mixtures, a short-lived stability may obtain. Great caution is necessary in adding antitoxin in small quantity to avoid overneutralization, even though tests, made after the mixture has been prepared, may indicate satisfactory balance. Furthermore, each time an additional quantity of antitoxin is added, the period required for complete combination with toxin is delayed. When finally the reaction of the mixture on guineapigs seems satisfactory, disappointment may result in that after a lapse of time the test guinea-pigs fail to die within the required period.

The calculation of the amount of antitoxin required for properly balancing a toxin-antitoxin mixture is acconpanied by several complications. If the number of antitoxic units and $\mathrm{L}+$ doses of toxin is the same, one M L D for each $\mathrm{L}+$ dose should theoretically remain unneutralized and the mixture would be far too toxic. This obviously can but rarely be true for several reasons. The most important one of these reasons is the fact that an exact unitage is usually not determined. The common practice is not to determine the potency of a serum closer than 50 or 25 units apart. This would, for example, mean that an 800 unit serum actually may contain 800 to 849 or 800 to 824 units. A second complication lies in the varying susceptibility of guinea-pigs. While it may be permissible to consider the potency of a serum ascertained when the guinea-pigs barely survive the 96-hour period, as a rule, the value is taken from guinea-pigs surviving a variable period beyond 96 hours. The serum therefore in reality contains a distinct excess of potency over the claimed value. Finally, there is some uncertainty as to the actual value of the antitoxin, since the original standard of Ehrlich has been changed. While Ehrlich's standard called for the control guinea-pig's death in 4 days, the new standard calls for its death in 3 days. The latter standard leaves, 
therefore, an uncertain excess of potency in the antitoxin, which is a wise measure for safety, but upsets calculations somewhat, because it is not known whether a definite relation exists between the amount of toxin necessary to kill a guinea-pig in three days and the amount necessary to kill in 4 days. As a matter of fact, an exceedingly large amount of toxin rarely kills a guinea-pig in less than 36 to 40 hours. It seems almost unnecessary to add that by using a low potency antitoxin for toxin-antitoxin mixtures the required quantity can be calculated and measured with less chance of overneutralization than by using a high unit antitoxin.

TABLE 1.

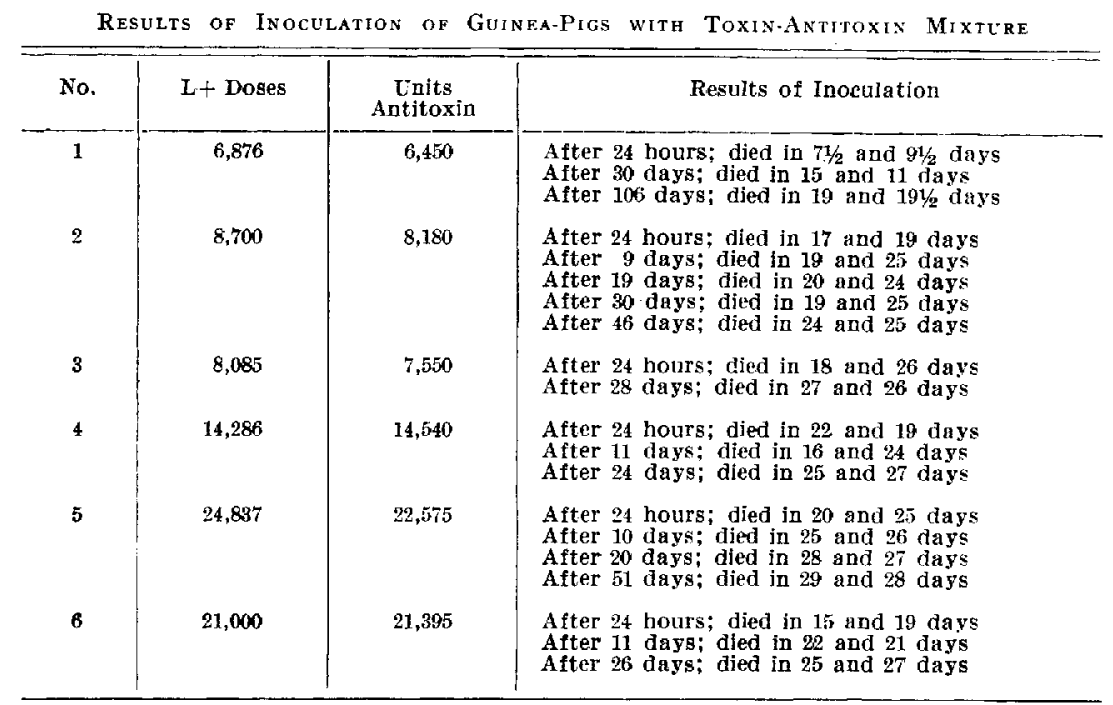

Table 1 gives the amounts of $\mathrm{L}+$ doses and antitoxic units of several selected lots of toxin-antitoxin mixture. The results of comprehensive tests of these lots have shown clearly that satisfactory mixtures were obtained. Dead guinea-pigs were always examined for toxin reactions and possible infections.

In some instances the number of $\mathrm{L}+$ doses apparently exceeded that of antitoxic tnits. Sometimes the difference is marked. In other cases the reverse is true. Since the same lot of antitoxin was used in all lots, it seems obvious that the variation must lie in the constitution of the toxin. The variation of quantitative relation between the number of antitoxic units and $\mathrm{L}+$ doses may be explained by assuming that 
the larger the prototoxoid group of the toxin molecule is, the larger is the amount of antitoxin required. In no case, however, is the number of antitoxic units as great as would be necessary were the units accurately determined, because there was an excess of potency in the antitoxin. In other words, the excess toxin, as shown by guinea-pig tests, is actually smaller than the relation of antitoxin to toxin suggests.

In the table appear the records of the guinea-pigs which received five human doses. The deaths of the guinea-pigs were somewhat delayed as the age of the mixture increased. The differences between these periods were not very large, but sufficiently so to suggest a slow combination between toxin and antitoxin. As the combining process proceeds death is deferred, disregarding a similar delay caused by gradual deterioration of both components.

One lot which is not recorded in the table had the effect of shortening the periods between injections and the death of the guinea-pigs. The results were: After 24 hours they died in $6 \frac{1}{2}$ and 7 days; after 9 days they died in 8 and $7 \frac{1}{2}$ days; after 22 days they died in 5 and 6 days; after 66 days both died in 4 days; after 73 days they died in 2 and 5 days; after 86 days they died in 2 and 3 days. This example is interesting because the second period was greater than the first, but after that the periods grow shorter.

An experiment was made by mixing 4 small lots, one of which did not possess sufficient toxicity to kill a guinea-pig when 5 human doses were injected. There was loss of weight for 10 days followed by increasing weight and recovery. The other 3 lots were fatal to guineapigs in 6,2 and 32 days, respectively. The mixture was tested after 5 days. The guinea-pigs died in 22 and 24 days; 11 days later after 24 and 19 days; 20 days later they died after 18 and 25 days; 25 days later they died in 20 and 22 days; 30 days later they died in 22 and 24 days; 35 days later they died in 21 and 30 days; 60 days later they died in 25 and 29 days. The mixture was fairly stable, but it is doubtful whether such mixtures will always yield dependable results.

After toxin and antitoxin have been mixed there are three possibilities conceivable which may influence the result. These possibilities are:

1. Both toxin and antitoxin deteriorate in approximately the same proportion.

2. The antitoxin deteriorates more rapidly than the toxin.

3 . The toxin deteriorates more rapidly than the antitoxin. 
Under conditions when antitoxin and toxin deteriorate in the same proportion a simple consideration shows what will happen. The total toxicity and the total antitoxin decrease as time progresses with a corresponding decrease of potency of the mixture. As it is known that both toxin and antitoxin deteriorate in time, a reasonable time limit is placed on the potency of toxin-antitoxin mixtures. The custom at present is to estimate this limit as six months.

The second condition, namely, the more rapid deterioration of antitoxin, is likely to occur rarely, since experience has shown that, as a rule, antitoxin is more stable than toxin. However, such a condition is conceivable and cannot be entirely excluded. The consequence would be an increasing relative toxicity of the mixture.

The third condition probably occurs more frequently than the previous two. When the toxin deteriorates more rapidly than the antitoxin the loss of potency of the mixture is relatively rapid.

Several lots of toxin-antitoxin mixture increased in toxicity as time advanced. An example has been mentioned in which the rate of increasing toxicity may not have been negligible. This puzzling phenomenon is difficult to explain, because the antitoxin used was of considerable age and had been tested several times in order to show its stability. The following explanation is offered as tentative; it may not pass the test of time, but may stimulate further investigation.

Ehrlich assumed that the toxin molecule consists of prototoxoid, toxin and toxone. It is not probable that any two toxins are composed of these portions in the same proportion. But does one lot of toxin always contain only one kind of molecules, or are there sometimes in the same lot molecules whose fractions differ in proportion? When antitoxin is added to toxin, it combines first with the prototoxoid group, next with the toxin group and lastly with the toxone group. This process takes time; how much, it would be venturesome to suggest, but probably the time is not the same in all cases, depending on the constitution of the toxin. Since antitoxin combines first with the prototoxoid group it would seem that the larger this group is the shorter would be the time required for combination.

If it is assumed that some toxins are not homogeneous, antitoxin would combine with the three groups, but on standing it would find some molecules with a larger prototoxoid group than the combined molecules possess. The antitoxin would then go over in part to the newly found molecules, leaving more toxin and toxone free. While this process is going on, the toxicity of the mixture increases. Under 
varying conditions the increase of toxicity might be relatively siow or rapid. This view is supported by some observations which seemed to show that portions taken from different layers of a mixture gave somewhat different results when tested on guinea-pigs. If the antitoxin combines with molecules of a small prototoxoid group first there is no change in toxicity.

Another possibility is suggested by the fact that toxin deteriorates. During this process the prototoxoid group may increase at the expense of the toxin and toxone fractions, involving a decrease of toxicity. If the enlargement of the prototoxoid group is proportionately greater than the deterioration of the toxin group, the toxicity of the mixture would increase, despite deterioration of the toxin group. In other words, some antitoxin combined with the toxin and toxone groups would pass over to molecules with increased prototoxoid. A condition of this kind becomes more complicated, since probably the rate of deterioration of toxin molecules does not progress at the same rate. However, the increase of toxicity would probably be relatively small.

It has been stated before that toxin should not be addled to compensate for overneutralization of a mixture. This might be permissible if the identical toxin were used, although there are no experimental data on this point. If a different toxin is added, the stability of the mixture would be materially injured by gradual movement of antitoxin from molecules with small prototoxoid to those with latge ones. This also, as a rule, would involve an increase of toxicity.

The theory suggested is based on the following assumptions: (1) the correctness of Ehrlich's theory of the constitution of the toxin molecule; ( 2 the greater avidity of antitoxin for prototoxoid than for toxin, and the greater avidity of antitoxin for toxin than for toxone; (3) the relative firmness of the combination between antitoxin and prototoxoid as compared with its combination with toxin and toxone; (4) at best, combination between toxin and antitoxin is a slow process when compared with chemical reactions, and (5) not all toxins are entirely homogeneous.

The theory seems to offer a tentative explanation of some puzzling phenomena observed in the action of toxin-antitoxin mixtures. The necessity of exerting the utmost care in the preparation of toxinantitoxin mixtures is emphasized by the fact that occasionally a mixture which has given apparently satisfactory tests later increases in toxicity. Toxins should be well ripened, and both toxin and antitoxin should be tested repeatedly until the values obtained are fairly uniform. With 
care the toxicity probably will never increase to a point at which it becomes really dangerous, but local and systemic reactions may be severe if the excess toxin is too large or becomes so after standing. The greatest aid in obtaining a safe preparation is time. The longer the period of test-within reasonable limits-the more dependable, as a rule, is the product. 